\title{
Compact object mergers: observations of supermassive binary black holes and stellar tidal disruption events
}

\author{
S. Komossa and J. A. Zensus \\ Max-Planck-Institut für Radioastronomie, Auf dem Hügel 69, 53121 Bonn, Germany \\ email: skomossa@mpifr.de
}

\begin{abstract}
The capture and disruption of stars by supermassive black holes (SMBHs), and the formation and coalescence of binaries, are inevitable consequences of the presence of SMBHs at the cores of galaxies. Pairs of active galactic nuclei (AGN) and binary SMBHs are important stages in the evolution of galaxy mergers, and an intense search for these systems is currently ongoing. In the early and advanced stages of galaxy merging, observations of the triggering of accretion onto one or both $\mathrm{BHs}$ inform us about feedback processes and $\mathrm{BH}$ growth. Identification of the compact binary SMBHs at parsec and sub-parsec scales provides us with important constraints on the interaction processes that govern the shrinkage of the binary beyond the "final parsec". Coalescing binary SMBHs are among the most powerful sources of gravitational waves (GWs) in the universe. Stellar tidal disruption events (TDEs) appear as luminous, transient, accretion flares when part of the stellar material is accreted by the SMBH. About 30 events have been identified by multi-wavelength observations by now, and they will be detected in the thousands in future ground-based or space-based transient surveys. The study of TDEs provides us with a variety of new astrophysical tools and applications, related to fundamental physics or astrophysics. Here, we provide a review of the current status of observations of SMBH pairs and binaries, and TDEs, and discuss astrophysical implications.
\end{abstract}

Keywords. Black holes, galaxies, mergers, accretion, jets

\section{Introduction: galaxy mergers and supermassive binary black holes}

Galaxies have merged frequently with each other throughout the history of the universe. Galaxy mergers trigger quasars, are the sites of major black hole growth, and are believed to fix the scaling relations between SMBH mass and host properties - either by merging repeatedly with each other, and/or by feedback processes following the onset of accretion onto one or both black holes. If both galaxies harbor SMBHs at their centers, these two will ultimately form a bound pair. Coalescing supermassive binary black holes (SMBBHs) are among the most powerful emitters of gravitational waves in the universe. The subsequent gravitational wave recoil of the newly formed single black hole will, in rare cases, lead to kick velocities exceeding the host's escape velocity, and astrophysical consequences of this phenomenon are now being explored.

Galaxy and SMBH mergers evolve in several stages (e.g. Begelman et al. 1980; Roos 1981; Merritt \& Milosavljević 2005; Colpi 2014; our Fig. 1). During the first stage, merging of the two galaxies is dominated by dynamical friction. At separations on the order of parsecs, the two BHs form a bound pair. That binary then hardens by interactions with stars and gas. The efficiency of these processes in shrinking the binary orbit has been much discussed in the literature, and is known as the "final-parsec problem", reflecting early results and concerns that the binary may stall at parsec-scale separations for more than a Hubble time, rarely reaching a regime where efficient GW emission leads to 


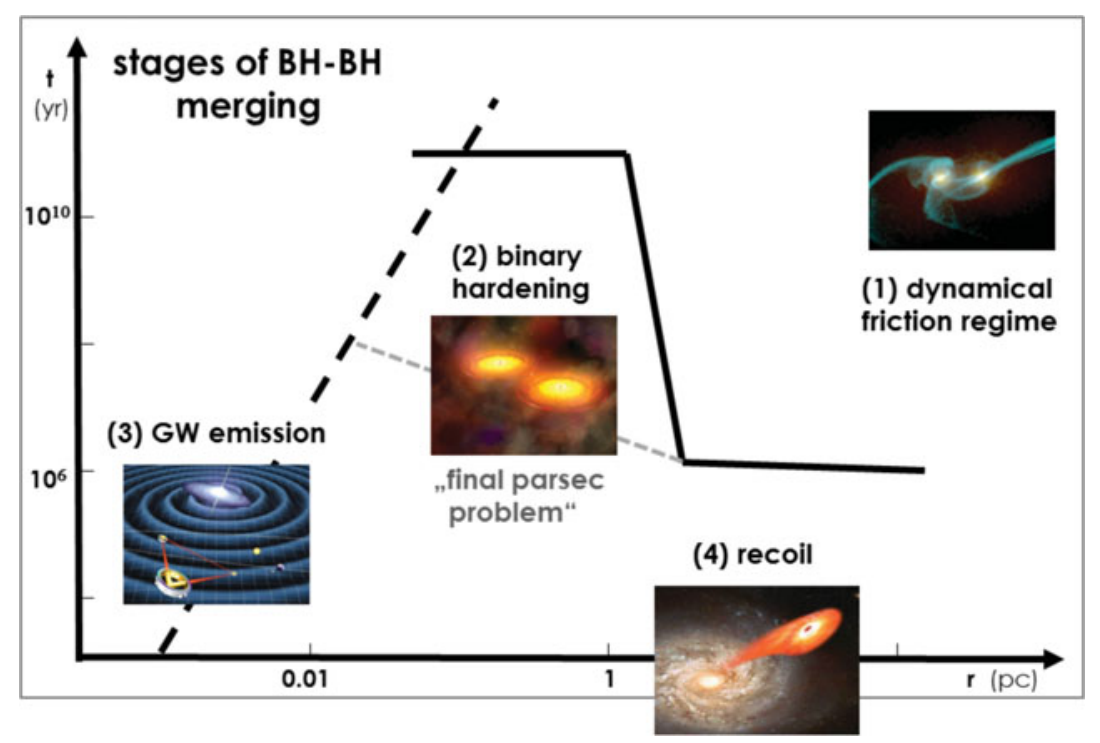

Figure 1. Stages of the evolution of SMBH pairs in the course of galaxy merging (following Begelman et al. 1980).

rapid coalescence of the system. Recent results indicate that non-axisymmetric galaxy potentials, the abundance of stars with centrophilic orbits, and/or the presence of large amounts of gas are, in many cases, sufficient to shrink the orbit in less than a Hubble time (e.g. Perets \& Alexander 2008; Preto et al. 2011; Khan et al. 2013; Mayer 2013; Chapon et al. 2013; Ivanov et al. 2015; Aly et al. 2015; Vasiliev 2015; review by Colpi 2014). At coalescence, the emitted GWs carry away linear momentum, so that the SMBH receives a kick velocity, and then oscillates about the core of its host galaxy, or in rare cases escapes (e.g. Campanelli et al. 2007; Lousto \& Zlochower 2011; review by Centrella et al. 2010).

Key questions related to all stages of galaxy merging include the following: (a) when does the accretion process start, (b) how long does it last, (c) how much matter is accreted before and after binary coalescence, (d) how much do the SMBHs grow in each phase, (e) how often are both SMBHs active, (f) how efficient are feedback processes, (g) how efficient is the loss of angular momentum due to interactions with gas and stars, (h) how much do the SMBHs' spins change during accretion, (i) how quick does the SMBBH coalesce, $(\mathrm{j})$ how frequent are coalescences in the universe, and $(\mathrm{k})$ what is the amplitude of GW recoil?

The answers to these questions are central to our understanding of the assembly history and demography of black holes, and of galaxy formation and evolution across cosmic times. Identifying SMBBHs in all stages of their evolution is therefore of great interest, and an intense search is currently ongoing.

A variety of signatures have been used to search for and identify pairs, SMBBHs, and candidates (Fig. 2). Detection of the wide systems, when the two black holes are spatially resolved from each other, is observationally most easy and robust. More indirect methods are in use to search for the closest SMBBH systems, no longer spatially resolved. Semiperiodicities in lightcurves or spatial structures in radio jets, double-peaked emission lines, and other features, have all been used to identify candidates. Most methods require that both, or at least one SMBH, is active. Most difficult to recognize are SMBBHs at the cores of non-active galaxies. They could be widely present, and with current methods 
(and with the single exception of our own Galactic Center), we would have almost no way of detecting them. A recent suggestion has therefore been to use the lightcurves of flares from tidally disrupted stars to search for the tell-tale signatures of binaries in otherwise quiescent galaxies (Sect. 3.3).

This review provides a short overview of observations of, and search strategies for, pairs and binary SMBHs at wide and close orbital separations. We will not cover the widest systems of AGN pairs, in early stages of interaction, or multiple AGN in clusters of galaxies, due to lack of space. An accompanying review (Liu 2015, these proceedings) will elaborate in much greater depth on theoretical aspects, and theoretical predictions of signatures of SMBBHs which have not yet been observed, but can be used for future searches. Further, this contribution will focus on main principles and detection methods, and a few prime representative systems. There is not enough space to reference all publications that have contributed to this exciting and rapidly growing field. Our apologies in advance.

\section{Spatially resolved systems in single galaxies and advanced mergers}

Wide pairs of accreting SMBHs, spatially resolved, can be identified by the characteristic signatures of AGN activity from both $\mathrm{BHs}$, in form of luminous (hard) X-ray emission, compact radio cores, typical optical emission-line ratios, or IR colours. Only a few systems have been identified at projected separations of $r \sim 1 \mathrm{kpc}$ or less. In X-rays, these are NGC 6240 (at $r=1 \mathrm{kpc}$; Komossa et al. 2003) and NGC 3393 (at $r=150$ pc; Fabbiano et al. 2011), both based on high-resolution Chandra imaging spectroscopy. In the radio regime, two compact, variable, flat-spectrum cores were found in 0402+379 (at $r=7$ pc; Rodriguez et al. 2006; Burke-Spolaor 2011). In the optical band, two candidate AGN cores exist in SDSSJ132323.33-15941.9 (at $r=0.8 \mathrm{kpc}$; Woo et al. 2014).

The galaxy pair SDSSJ1502+1115 (Sect. 3.1) is remarkable for its overall radio structure. It consists of two bright radio cores at $7.4 \mathrm{kpc}$ separation (Fu et al. 2011b). One of the two is further resolved into two knots of about equal brightness at 140 pc projected separation. These have been interpreted as representing either two separate SMBHs, or else double hot spots around a single SMBH (Deane et al. 2014; Wrobel et al. 2014).

\section{Candidate spatially unresolved systems}

\subsection{Double-peaked emission lines}

Optical spectra of AGN are characterized by narrow and broad emission lines. If these appear double, they may indicate the presence of two accreting SMBHs (Gaskell 1983; 1996; Zhou et al. 2004; review by Popović 2012). Further, single-peaked emission lines, which are kinematically shifted with respect to their host galaxy, may imply the presence of a merger (Comerford et al. 2009).

In recent years, larger samples of AGN with doubled-peaked narrow lines ("narrow-line double-peakers") have been identified thanks to large spectroscopic surveys like SDSS, AGES and LAMOST (e.g. Wang et al. 2009; Komossa \& Xu 2009; Liu et al. 2010; Smith et al. 2010; Ge et al. 2012; Comerford et al. 2013; Barrows et al. 2013; Shi et al. 2014). A challenge when identifying the true binary AGN among them arises from the fact, that several other mechanisms do exist, which also produce double-peaked lines, but only involve a single AGN. These include the presence of two-sided jets or outflows, rotating disks, or a single AGN which ionizes the interstellar media of two host galaxies (e.g. $\mathrm{Xu} \&$ Komossa 2009). Further, double-peakers are only expected for a short fraction of 


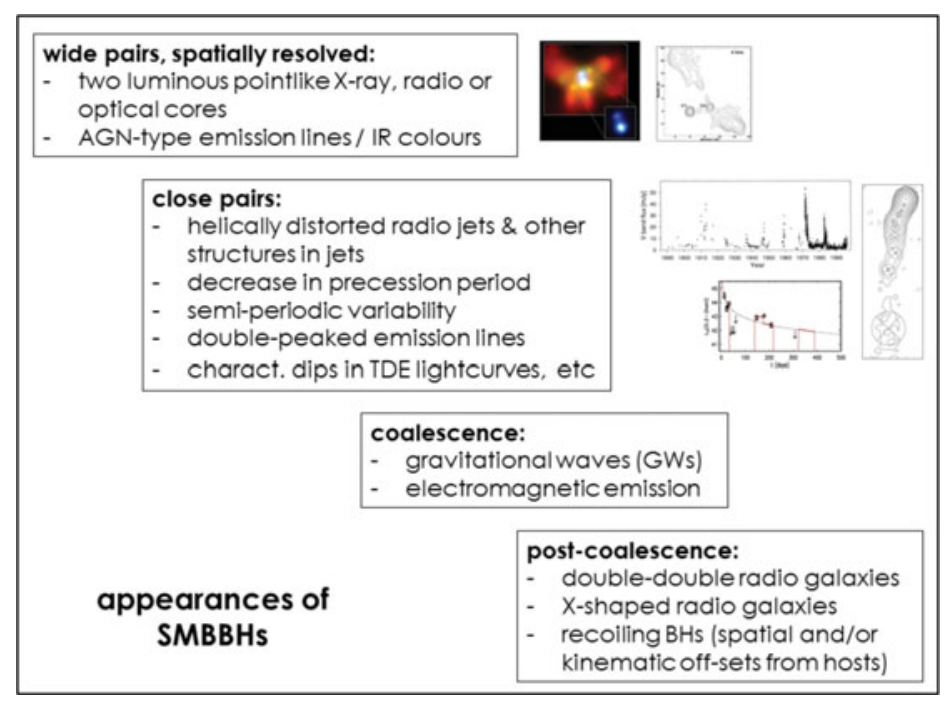

Figure 2. Signatures and detection methods of SMBH pairs and binaries.

the total merger time (Yu et al. 2011, van Wassenhove et al. 2012; Blecha et al. 2013). Therefore, multi-wavelength follow-up observations are required, in order to select the binaries among the large numbers of double-peakers. Such follow-ups have shown that only a small fraction of them, $\sim 2 \%-10 \%$, harbor AGN pairs (e.g. Fu et al. 2011a; Fu et al. 2012; Shen et al. 2011; Smith et al. 2012; Comerford et al. 2012). One of the confirmed systems is SDSSJ1502+1115, with two luminous radio cores at a projected spatial separation of $7.4 \mathrm{kpc}(\mathrm{Fu}$ et al. $2011 \mathrm{~b}) . \dagger$

A fraction of all quasar spectra exhibits double-peaked broad emission lines. If these are due to two broad-line regions bound to two SMBHs orbiting each other, we should see the characteristic Doppler-shifts of the emission lines reflecting the orbital motion (Gaskell 1983; Shen \& Loeb 2010). Broad-line double-peakers carefully monitored in the 1980s and 90s (e.g. Halpern \& Filippenko 1988; Halpern \& Eracleous 2000) did not reveal the expected orbital motions, and have been interpreted as systems with warped accretion disks around single SMBHs instead. New large samples of broad-line double-peakers, or of systems with single, kinematically shifted broad lines, have now been selected from SDSS (e.g. Tsalmantza et al. 2011; Eracleous et al. 2012; Decarli et al. 2013; Shen et al. 2013; Ju et al. 2013), and some binaries may hide among them.

Recently, Bon et al. (2012) presented a SMBBH model for the well-known, nearby, broad-line Seyfert galaxy NGC 4151, based on evidence for periodic variations of the observed $\mathrm{H} \alpha$ emission line, in many years of spectroscopic monitoring. The observations have been explained with a sub-parsec binary with an orbital period of $\sim 16 \mathrm{yr}$.

\subsection{Semi-periodic variability}

. A number of blazars show evidence for semi-periodic optical variability, which might be linked to the presence of a second SMBH (e.g. Sillanpää et al. 1988; Raiteri et al. 2001, Fan et al. 2002; de Paolis et al. 2002; Rieger 2004; Ostorero et al. 2004; Liu et al. 2006;

$\dagger$ Note that we list narrow double-peakers under the Section of "unresolved sources", because their initial selection criterion is (in most cases) based on spatially unresolved emission. Follow-up imaging, when available, then often did resolve the sources. However, they usually consist of wider pairs of galaxies with core separations above a kpc, not fitting in the category discussed in Section 2. 
Qian et al. 2007; Volvach et al. 2007; Xie et al. 2008; Karouzos et al. 2010; Kudryavtseva et al. 2011; Graham et al. 2015). The best studied such case is the blazar OJ287. Its optical lightcurve has been observed for more than a century (thanks to photographic plate archives all around the world, and intense dedicated monitoring during the last few decades) and shows repeat outbursts every $\sim 12$ yr (e.g. Sillanpää et al. 1988; Valtaoja et al. 2000, Valtonen et al. 2012), each of which is composed of two peaks separated by 1 yr. The best explored SMBBH model consists of a secondary BH in a precessing orbit, which impacts a warped accretion disk around the primary twice each orbit. Precise timing of the past optical peaks then allows to derive the orbital parameters of the system. Calculating Keplerian orbits with post-Newtonian corrections, Valtonen (2007) presented an orbital solution with a primary mass of $\sim 2 \times 10^{10} \mathrm{M}_{\odot}$, a mass ratio $\sim 0.01$, an eccentricity of $\epsilon=0.66$, and a semi-major axis of $0.045 \mathrm{pc}$. They also reported tentative evidence for an orbital shrinkage due to emission of GWs, of order $\Delta T_{\mathrm{GW}} \approx 0.01$ yr/period (Valtonen et al. 2008). The next optical maximum is expected in a few years, allowing to test new predictions of all recent models for OJ287.

\subsection{Dips in TDE lightcurves}

MBBHs imprint their presence on the outburst lightcurves of stellar tidal disruption events (TDEs). The secondary temporary interrupts the accretion stream on the primary, causing characteristic deep dips in the decline lightcurves (Liu et al. 2009). This signature has been observed in the lightcurve of the TDE from SDSSJ120136.02+300305.5, which is well modelled with a binary of mass ratio $q \sim 0.1$ at $\sim 0.6 \mathrm{mpc}$ spatial separation (Liu et al. 2014). In the future, SMBBHs with TDEs may also be recognized by reprocessed emission lines which will show a tilted response function due to the off-centre location of one of the SMBHs (Brem et al. 2014).

\subsection{Structures in radio jets}

Several blazar radio jets show semi-periodic deviations from a straight line, and/or some other unusual structures. One way to explain these observations is involving the presence of a binary SMBH, which causes either (1) a modulation due to orbital motion of the jet-emitting $\mathrm{BH}$ around the primary $\mathrm{BH}$, or (2) jet precession (e.g. Begelman et al. 1980; Roos 1988; Hardee et al. 1994, Britzen et al. 2001) †. If the jet precession is caused by a binary, then a prediction of this scenario is the acceleration of jet precession, observable on long timescales (Liu \& Chen 2007).

Radio interferometry has provided us with the highest-resolution observations of jets over decades. Here, we would like to mention three representative candidate SMBBH systems. These are among the well-studied systems, but there is a number of others which would deserve mentioning, and are not due to lack of space. The quasar S5 1928+738 has long been suspected to harbor a SMBBH (Hummel et al. 1992; Roos et al. 1993; Murphy et al. 2003; see also Roland et al. 2015). Kun et al. (2014), analyzing $20 \mathrm{yr}$ of VLBI data, presented evidence that the jet-emitting SMBH is actually spinning. Their orbital modelling implies a binary separation of $\sim 10 \mathrm{mpc}$, and an orbital period of $\sim 5 \mathrm{yr}$. The helical distortions of the jet of the BL Lac object Mrk 501 have been interpreted with a SMBBH model by Conway \& Wrobel (1995) and Villata \& Raiteri (1999). A SMBBH scenario was also involved in order to explain evidence for semi-periodic variability of this source (Rieger \& Manheim 2000; see also de Paolis et al. 2002; Rödig et al. 2009). Lobanov \& Roland (2005) presented a SMBBH model at $~ 0.3$ pc separation for the quasar 3C345,

$\dagger$ See, e.g. Britzen et al. 2010; Lobanov \& Roland 2005, and Godfrey et al. 2012 (and references therein), for a discussion including alternative scenarios such as disk oscillations or Kelvin-Helmholtz instabilities. 
which can reproduce both, its optical and radio variability, and the morphology and kinematics of the parsec-scale jet.

The most powerful method to date of spatially resolving the orbit of a jet-emitting $\mathrm{BH}$ in a compact binary is phase-referencing of VLBI radio data. Using that technique, Sudou et al. (2003) reported evidence for systematic changes in radio position, which they interpreted as orbital motion of the radio core of $3 \mathrm{C} 66 \mathrm{~B}$ with a period of $1.05 \mathrm{yr}$. Part of the possible orbital solutions could be excluded based on current pulsar timing constraints (Jenet et al. 2004), while the rest remains a possibility (Iguchi et al. 2010). Future phase-reference measurements of this and other systems, along with simulations of the jet base, core-shift measurements, and studies of transverse motions will provide us with strong tests of the SMBBH model.

\section{Post-coalescence candidates}

Certain signatures of compact and coalescing binaries remain imprinted on their largescale environment, and can therefore be recovered from multi-wavelength observations long after the actual coalescence. For instance, accretion temporarily interrupts in compact binaries, because of the fast orbital shrinkage due to GW emission, dominating over viscous processes, so that the inner disk no longer catches up (e.g. Liu et al. 2003; Milosavljević \& Phinney 2005; Farris et al. 2015). If these systems launch radio jets, jet formation will be temporarily interrupted, too, and this may explain the presence of double-double radio galaxies (Liu et al. 2003). If the hole's spin direction changes after coalescence, the jet will be launched in a new direction, and this is one possibility to account for the structure of X-shaped radio galaxies (Merritt \& Ekers 2002; see GopalKrishna et al. 2012 for a recent overview; see also Mezcua et al. 2012). If the newly formed single SMBH receives a significant kick velocity after coalescence, it will appear spatially or kinematically off-set from its host galaxy, and several candidate recoiling SMBHs have emerged in recent years (review by Komossa 2012a). Further, it has been suggested that the central stellar light deficits observed in some ellipticals and bulges were created by SMBBHs which had shrunk their orbits by slingshot ejection of stars, consistent with recent observations (e.g. Dullo \& Graham 2014).

\section{Future missions and searches}

A number of ongoing and future missions and surveys will be sensitive to SMBBHs in all stages of evolution. For instance, space VLBI and mm VLBI at the shortest wavelengths feasible will provide us with the highest spatial resolution (e.g. Fish et al. 2013; Tilanus et al. 2014), while the Square Kilometer Array (SKA) will provide high sensitivity (e.g. Deane et al. 2015). SKA and other current and upcoming PTA (pulsar timing array) experiments will detect the gravitational wave signatures of the most massive coalescing SMBBHs using pulsar timing (e.g. Lazio 2013; Hobbs 2013; Kramer \& Champion 2013; Sesana 2015). Future high-sensitivity X-ray observatories may kinematically resolve binary effects on the iron line profile from one or two disks (Yu \& Lu 2001; McKernan et al. 2013; Jovanović et al. 2014), while optical integral field spectroscopy may reveal the kinematic signature of the inspiral phase (Meiron \& Laor 2013).

Further breakthroughs in the field are expected once space-based gravitational-wave interferometers are in operation, providing measurements of coalescence rates, SMBH masses and spins (e.g. Babak et al. 2011; review by Barausse et al. 2015). eLISA is currently scheduled for launch around 2030. Electromagnetic counterparts to GWs (e.g. 
Schutz 1986) from coalescing SMBBHs, and characteristic signals before or after coalescence, may appear as transients in current or future transient surveys (reviews by Schnittman 2011; Haiman 2012).

\section{Tidal disruption of stars by supermassive black holes}

The tidal disruption, and subsequent accretion, of a star by a supermassive black hole produces a luminous flare of electromagnetic radiation (e.g. Rees 1990; Luminet 1985). A star is disrupted, once the tidal forces of the hole exceed the self-gravity of the star (Hills 1975). The distance at which this happens, the tidal radius, is given by

$$
r_{\mathrm{t}} \simeq 7 \times 10^{12}\left(\frac{M_{\mathrm{BH}}}{10^{6} \mathrm{M}_{\odot}}\right)^{\frac{1}{3}}\left(\frac{M_{*}}{\mathrm{M}_{\odot}}\right)^{-\frac{1}{3}}\left(\frac{r_{*}}{\mathrm{R}_{\odot}}\right) \mathrm{cm}
$$

A fraction of the stellar material will be on unbound orbits and escape, while the rest will eventually be accreted (Fig. 3). The events appear as luminous transients with peak in the UV or soft X-rays, declining on the timescale of months to years (e.g. Rees 1990; Evans \& Kochanek 1989). Recent state of the art modelling has addressed the different stages of TDE evolution under various conditions (e.g. Lodato et al. 2009; Brassart \& Luminet 2010; Strubbe \& Quataert 2011; Lodato \& Rossi 2011; Cheng et al. 2012; Kesden 2012; Guillochon \& Ramirez-Ruiz 2013; Hayasaki et al. 2013, Dai \& Blandford 2013; Cheng \& Bogdanovic 2014; Shiokawa et al. 2015; and references therein). If the doomed star is compact (e.g. a white dwarf), partial disruption will produce an electromagnetic and a GW signal (review by Amaro-Seoane et al. 2007).

Observing TDEs, out to large cosmic distances, provides us with a variety of new astrophysical tools and applications, related to fundamental physics or astrophysics, including studying precession effects in the Kerr metric, measuring BH spin, observing other relativistic effects (at $10^{8} \mathrm{M}_{\odot}$, the tidal radius is on the order of the Schwarzschild radius), probing accretion physics under extreme conditions and near $L_{\text {edd }}$, understanding the physics of jet formation and early evolution, reverberation-mapping the gaseous core environment via its emission-line response, searching for a population of (so far elusive) intermediate mass BHs, detecting supermassive binary BHs at the cores of quiescent galaxies (from TDE lightcurves; Sect. 3.3), probing stellar kinematics on spatial scales which cannot be resolved directly (via disruption rates in different types of galaxies), or spotting recoiling BHs by off-nuclear TDEs.

\section{Multi-wavelength observations}

A key signpost of TDEs is their luminous, transient high-energy emission, peaking in the UV or soft X-rays, arising from the accretion of the stellar material. First events from quiescent galaxies have been identified in the course of the ROSAT all sky survey, which was ideal for detection because of its repeat coverage of almost the whole sky, and its high sensitivity in the soft X-ray band (0.1-2.4 keV). Events appeared as luminous transients, reaching peak luminosities up to $>10^{43-44} \mathrm{erg} \mathrm{s}^{-1}$ just in soft X-rays (e.g. Bade et al. 1996; Komossa \& Bade 1999; Grupe et al. 1999). They then faded away by factors larger than a few thousand (Halpern et al. 2004; Komossa et al. 2004), their initially supersoft Xray spectra $(k T \sim 0.04-0.1 \mathrm{keV})$ showed a hardening with time, and optical spectroscopy of the host galaxies revealed little or no activity at all (review by Komossa 2002). Two of these events, NGC 5905 and RXJ1242-1119, continue to be the best-monitored events in terms of their long-term X-ray lightcurves, spanning time intervals of more than a decade 


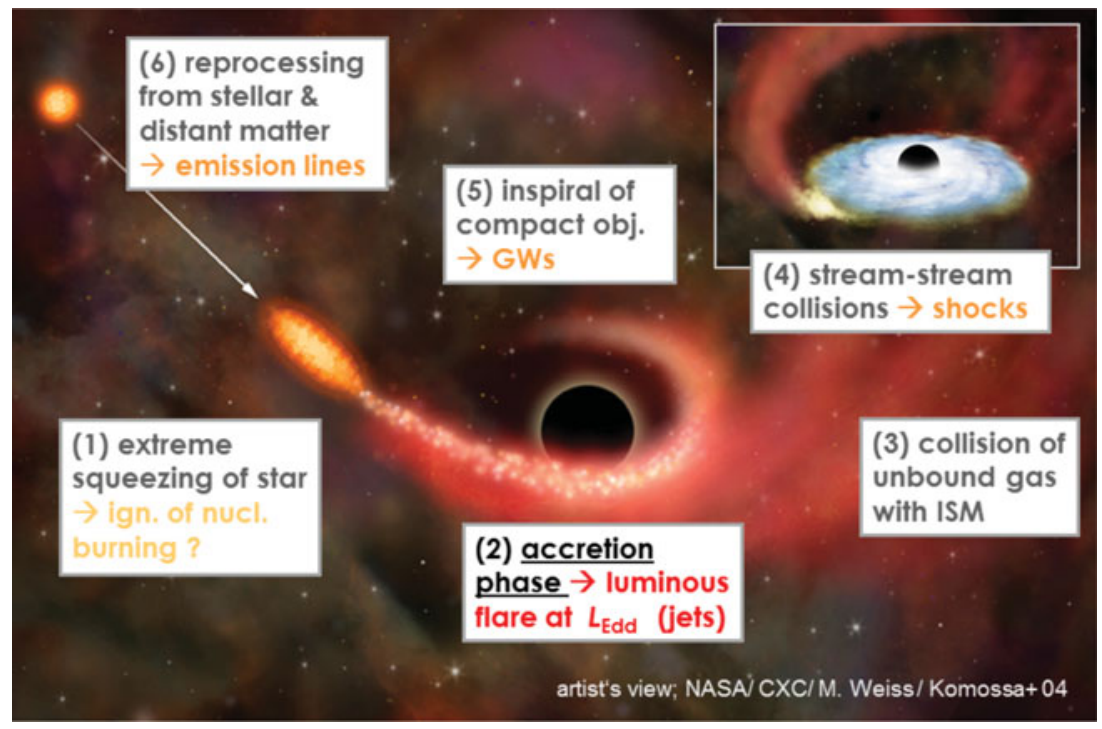

Figure 3. Evolution of stellar tidal disruption events and sources of radiation. In most cases, the accretion phase is the most luminous electromagnetic phase.

(Komossa et al. 2004, Halpern et al. 2004). All the event properties agree very well with order-of-magnitude predictions from tidal disruption theory (e.g. Rees 1988; 1990). More recently, in X-rays similar events have been found with Chandra and XMM-Newton (e.g. Esquej et al. 2008; Maksym et al. 2010; Lin et al. 2011; Saxton et al. 2012; Nikolajuk \& Walter 2013; Maksym et al. 2013; Donato et al. 2014), some of them with well-covered lightcurves during the first few years. Events were also found at longer wavelengths, in the UV and optical (e.g. Gezari et al. 2006; Komossa et al. 2008; van Velzen et al. 2011; Cenko et al. 2012a; Gezari et al. 2012; Chornock et al. 2014), some of them caught before their peak (see Komossa 2012b for a more extended review of multi- $\lambda$ observations). Several estimates of TDE rates are all on the order of $10^{-4}-10^{-5} \mathrm{yr}^{-1}$ galaxy $^{-1}$ (e.g. Donley et al. 2002; Esquej et al. 2008; Maksym et al. 2010; Wang et al. 2012) and agree well with theoretical predictions (e.g. Brockamp et al. 2011).

\section{Emission-line transients}

TDEs which occur in gas-rich galaxies will provide us with a powerful new tool of performing reverberation mapping of the cores of these galaxies. As the luminous electromagnetic radiation travels across the galaxy core, it will photoionize any circum-nuclear material (including the tidal debris itself) and is reprocessed into line radiation. Recently, SDSS and other surveys have enabled the discovery of several well-observed cases of transient optical emission lines, of a kind not observed before, and arising from otherwise quiescent galaxies $\uparrow$ : All of them exhibit bright, broad, fading emission from Helium and/or Hydrogen (Komossa et al. 2008; 2009, Wang et al. 2011; 2012; Gezari et al. 2012;

$\dagger$ These emission-line transients are markedly different from the mild line variability seen in AGN, with two exceptions: (1) The AGN IC 3599; which underwent a high-amplitude X-ray outburst accompanied by a strong increase in its optical emission lines (Brandt et al. 1995; Grupe et al. 1995; Komossa \& Bade 1999), and (2) the AGN NGC 1097, which shows strong, broad, double-peaked Balmer lines which emerged abruptly (e.g. Storchi-Bergmann et al. 1995). The underlying mechanism remains unknown, but high-amplitude Narrow-line Seyfert 1 variability (only IC 3599), variants of accretion-disk instabilities, or a TDE have all been considered. 
Gaskell \& Rojas Lobos 2014; Holoien et al. 2014, Arcavi et al. 2014), while some of them show transient super-strong iron coronal lines in addition, up to ionization stages of $\mathrm{Fe}^{13+}$ (Komossa et al. 2008; 2009; Wang et al. 2011; 2012).

\section{Jetted TDEs}

The possibility that TDEs launch radio jets, came up with the detection of the first few X-ray TDEs with ROSAT. Dedicated follow-ups of NGC 5905 did not detect any radio emission from a jet, however (Komossa 2002).

Two events recently discovered with Swift, Swift J1644+57 and Swift J2058.4+0516, differ from previous TDEs, in the sense that they had much harder X-ray spectra, were accompanied by strong (beamed) radio emission, and exhibit some other remarkable properties (e.g. Burrows et al. 2011; Bloom et al. 2011; Zauderer et al. 2011; 2013; Levan et al. 2011; Cenko et al. 2012b). Swift J1644+57 was detected with Swift BAT in 2011. Its (isotropic) peak luminosity exceeded $10^{48} \mathrm{erg} \mathrm{s}^{-1}$. The X-ray lightcurve shows a general downward trend, on which rapid, high-amplitude variability is superposed, as fast as $100 \mathrm{~s}$. After $\sim 1.5 \mathrm{yr}$, the X-rays suddenly dropped by a large factor, and have remained faint so far. The host galaxy at redshift $z=0.35$ does not show signs of permanent optical AGN activity. The event is accompanied by unresolved and variable radio emission, which has been interpreted as the rapid onset of a powerful jet after stellar tidal disruption. The event has motivated a large number of follow-ups and theoretical studies (review by Komossa 2015; in prep.), with an emphasis on the question of jet launching under TDE conditions, and the role of magnetic fields (e.g. Tchekhovskoy et al. 2014).

These and future observations of jetted TDEs provide us with a completely new probe of the early phases of jet formation and evolution in an otherwise quiescent environment without past radio-AGN activity.

\section{Future missions and surveys}

TDEs will be detected in large numbers with future sky surveys, including in the radio with SKA (Donnarumma et al. 2015), in the optical with LSST (Gezari et al. 2009), in hard X-rays with LOFT (Rossi et al. 2015), and in soft X-rays with the proposed mission Einstein Probe (Yuan et al. 2015). Well-covered lightcurves will enable a wealth of new science, and X-rays will be sensitive to relativistic effects (Sect. 6).

\section{Acknowledgements}

SK would like to thank ISSI/Bern for supporting and hosting two workshops on "Unveiling multiple AGN activity in galaxy mergers", and the participants for many stimulating discussions. SK would also like to thank NAOC Beijing for their great hospitality and support over many years. Many thanks to S. Britzen, T. Krichbaum, A. Lobanov, and E. Ros for a critical reading of the manuscript and very useful comments.

\section{References}

Aly, H., Dehnen, W., Nixon, C., \& King, A. 2015, MNRAS, 449, 65

Amaro-Seoane, P., Gair, J. R., Freitag, M., et al. 2007, Classical and Quantum Gravity, 24, 113

Arcavi, I., Gal-Yam, A., Sullivan, M., et al. 2014, ApJ, 793, 38

Babak, S., Gair, J. R., Petiteau, A., \& Sesana, A. 2011, Classical and Quantum Gravity, 28, 114001

Bade, N., Komossa, S., \& Dahlem, M. 1996, A\& A, 309, L35 
Barausse, E., Bellovary, J., Berti, E., et al. 2015, Journal of Physics Conference Series, 610, 012001

Barrows, R. S., Sandberg Lacy, C. H., Kennefick, J., et al. 2013, ApJ, 769, 95

Begelman, M. C., Blandford, R. D., \& Rees, M. J. 1980, Nature, 287, 307

Blecha, L., Loeb, A., \& Narayan, R. 2013, MNRAS, 429, 2594

Bloom, J. S., Giannios, D., Metzger, B. D., et al. 2011, Science, 333, 203

Bon, E., Jovanović, P., Marziani, P., et al. 2012, ApJ, 759, 118

Brandt, W. N., Pounds, K. A., \& Fink, H. 1995, MNRAS, 273, L47

Brassart, M. \& Luminet, J.-P. 2010, A\&A, 511, A80

Brem, P., Cuadra, J., Amaro-Seoane, P., \& Komossa, S. 2014, ApJ, 792, 100

Britzen, S., Roland, J., Laskar, J., et al. 2001, A\&A, 374, 784

Britzen, S., Kudryavtseva, N. A., Witzel, A., et al. 2010, A\&A, 511, A57

Brockamp, M., Baumgardt, H., \& Kroupa, P. 2011, MNRAS, 418, 1308

Burke-Spolaor, S. 2011, MNRAS, 410, 2113

Burrows, D. N., Kennea, J. A., Ghisellini, G., et al. 2011, Nature, 476, 421

Campanelli, M., Lousto, C., Zlochower, Y., \& Merritt, D. 2007, ApJ, 659, L5

Cenko, S. B., Bloom, J. S., Kulkarni, S. R., et al. 2012a, MNRAS, 420, 2684

Cenko, S. B., Krimm, H. A., Horesh, A., et al. 2012b, ApJ, 753, 77

Centrella, J., Baker, J. G., Kelly, B. J., \& van Meter, J. R. 2010, Reviews of Modern Physics, 82,3069

Chapon, D., Mayer, L., \& Teyssier, R. 2013, MNRAS, 429, 3114

Cheng, K.-S., Chernyshov, D. O., Dogiel, V. A., et al. 2012, ApJ, 746, 116

Cheng, R. M. \& Bogdanović, T. 2014, Phys. Rev. D, 90, 064020

Chornock, R., Berger, E., Gezari, S., et al. 2014, ApJ, 780, 44

Colpi, M. 2014, Space Sci. Rev., 183, 189

Comerford, J. M., Gerke, B. F., Stern, D., et al. 2012, ApJ, 753, 42

Comerford, J. M., Schluns, K., Greene, J. E., \& Cool, R. J. 2013, ApJ, 777, 64

Comerford, J. M., Gerke, B. F., Newman, J. A., et al. 2009, ApJ, 698, 956

Conway, J. E. \& Wrobel, J. M. 1995, ApJ, 439, 98

Dai, L. \& Blandford, R. 2013, MNRAS, 434, 2948

De Paolis, F., Ingrosso, G., \& Nucita, A. A. 2002, A\& A, 388, 470

Deane, R., Paragi, Z., Jarvis, M., et al. 2015, Advancing Astrophysics with the Square Kilometre Array (AASKA14), 151

Deane, R. P., Paragi, Z., Jarvis, M. J., et al. 2014, Nature, 511, 57

Decarli, R., Dotti, M., Fumagalli, M., et al. 2013, MNRAS, 433, 1492

Donato, D., Cenko, S. B., Covino, S., et al. 2014, ApJ, 781, 59

Donley, J. L., Brandt, W. N., Eracleous, M., \& Boller, T. 2002, AJ, 124, 1308

Donnarumma, I., Rossi, E. M., Fender, R., et al. 2015, Advancing Astrophysics with the Square Kilometre Array (AASKA14), 54

Dullo, B. T. \& Graham, A. W. 2014, MNRAS, 444, 2700

Eracleous, M., Boroson, T. A., Halpern, J. P., \& Liu, J. 2012, ApJS, 201, 23

Esquej, P., Saxton, R. D., Komossa, S., et al. 2008, A\&A, 489, 543

Evans, C. R. \& Kochanek, C. S. 1989, ApJ, 346, L13

Fabbiano, G., Wang, J., Elvis, M., \& Risaliti, G. 2011, Nature, 477, 431

Fan, J. H., Lin, R. G., Xie, G. Z., et al. 2002, A\& $A, 381,1$

Farris, B. D., Duffell, P., MacFadyen, A. I., \& Haiman, Z. 2015, MNRAS, 447, L80

Fish, V., Alef, W., Anderson, J., et al. 2013, ArXiv e-prints, arXiv:1309.3519

Fu, H., Myers, A. D., Djorgovski, S. G., \& Yan, L. 2011a, ApJ, 733, 103

Fu, H., Yan, L., Myers, A. D., et al. 2012, ApJ, 745, 67

Fu, H., Zhang, Z.-Y., Assef, R. J., et al. 2011b, ApJ, 740, L44

Gaskell, C. M. 1996, ApJ, 464, L107

Gaskell, C. M. \& Rojas Lobos, P. A. 2014, MNRAS, 438, L36

Gaskell, M. 1983, in Proceedings of the 24th Liege Int. Astrophys. Coll., 473

Ge, J.-Q., Hu, C., Wang, J.-M., Bai, J.-M., \& Zhang, S. 2012, ApJS, 201, 31 
Gezari, S., Martin, D. C., Milliard, B., et al. 2006, ApJ, 653, L25

Gezari, S., Strubbe, L., Bloom, J. S., et al. 2009, in ArXiv Astrophysics e-prints, Vol. 2010, astro2010: The Astronomy and Astrophysics Decadal Survey, 88

Gezari, S., Chornock, R., Rest, A., et al. 2012, Nature, 485, 217

Godfrey, L. E. H., Lovell, J. E. J., Burke-Spolaor, S., et al. 2012, ApJ, 758, L27

Gopal-Krishna, Biermann, P. L., Gergely, L. Á., \& Wiita, P. J. 2012, Research in Astronomy and Astrophysics, 12, 127

Graham, M. J., Djorgovski, S. G., Stern, D., et al. 2015, Nature, 518, 74

Grupe, D., Beuermann, K., Mannheim, K., et al. 1995, A\&A, 299, L5

Grupe, D., Thomas, H.-C., \& Leighly, K. M. 1999, A\&A, 350, L31

Guillochon, J. \& Ramirez-Ruiz, E. 2013, ApJ, 767, 25

Haiman, Z. 2012, in American Astronomical Society Meeting Abstracts, Vol. 220, American Astronomical Society Meeting Abstracts \#220, \#502.04

Halpern, J. P. \& Eracleous, M. 2000, ApJ, 531, 647

Halpern, J. P. \& Filippenko, A. V. 1988, Nature, 331, 46

Halpern, J. P., Gezari, S., \& Komossa, S. 2004, ApJ, 604, 572

Hardee, P. E., Cooper, M. A., \& Clarke, D. A. 1994, ApJ, 424, 126

Hayasaki, K., Stone, N., \& Loeb, A. 2013, MNRAS, 434, 909

Hills, J. G. 1975, Nature, 254, 295

Hobbs, G. 2013, in IAU Symposium, Vol. 291, IAU Symposium, ed. J. van Leeuwen, 165-170

Holoien, T. W.-S., Prieto, J. L., Bersier, D., et al. 2014, MNRAS, 445, 3263

Hummel, C. A., Schalinski, C. J., Krichbaum, T. P., et al. 1992, A\& A, 257, 489

Iguchi, S., Okuda, T., \& Sudou, H. 2010, ApJ, 724, L166

Ivanov, P. B., Papaloizou, J. C. B., Paardekooper, S.-J., \& Polnarev, A. G. 2015, A\& A, 576, A29

Jenet, F. A., Lommen, A., Larson, S. L., \& Wen, L. 2004, ApJ, 606, 799

Jovanović, P., Borka Jovanović, V., Borka, D., \& Bogdanović, T. 2014, Advances in Space Research, 54, 1448

Ju, W., Greene, J. E., Rafikov, R. R., Bickerton, S. J., \& Badenes, C. 2013, ApJ, 777, 44

Karouzos, M., Britzen, S., Eckart, A., Witzel, A., \& Zensus, A. 2010, A\&A, 519, A62

Kesden, M. 2012, Phys. Rev. D, 86, 064026

Khan, F. M., Holley-Bockelmann, K., Berczik, P., \& Just, A. 2013, ApJ, 773, 100

Komossa, S. 2002, in Reviews in Modern Astronomy, Vol. 15, Reviews in Modern Astronomy, ed. R. E. Schielicke, 27

Komossa, S. 2012 a, Advances in Astronomy, 2012, 14, (id. 364973)

Komossa, S. 2012b, in European Physical Journal Web of Conferences, Vol. 39, European Physical Journal Web of Conferences, 2001

Komossa, S. \& Bade, N. 1999, A\&A, 343, 775

Komossa, S., Burwitz, V., Hasinger, G., et al. 2003, ApJ, 582, L15

Komossa, S., Halpern, J., Schartel, N., et al. 2004, ApJ, 603, L17

Komossa, S., Zhou, H., Wang, T., et al. 2008, ApJ, 678, L13

Komossa, S., Zhou, H., Rau, A., et al. 2009, ApJ, 701, 105

Kramer, M. \& Champion, D. J. 2013, Classical and Quantum Gravity, 30, 224009

Kudryavtseva, N. A., Britzen, S., Witzel, A., et al. 2011, A\& A, 526, A51

Kun, E., Gabányi, K. É., Karouzos, M., Britzen, S., \& Gergely, L. Á. 2014, MNRAS, 445, 1370

Lazio, T. J. W. 2013, Classical and Quantum Gravity, 30, 224011

Levan, A. J., Tanvir, N. R., Cenko, S. B., et al. 2011, Science, 333, 199

Lin, D., Carrasco, E. R., Grupe, D., et al. 2011, ApJ, 738, 52

Liu, F. K. \& Chen, X. 2007, ApJ, 671, 1272

Liu, F. K., Li, S., \& Chen, X. 2009, ApJ, 706, L133

Liu, F. K., Li, S., \& Komossa, S. 2014, ApJ, 786, 103

Liu, F. K., Wu, X.-B., \& Cao, S. L. 2003, MNRAS, 340, 411

Liu, F. K., Zhao, G., \& Wu, X.-B. 2006, ApJ, 650, 749

Liu, X., Shen, Y., Strauss, M. A., \& Greene, J. E. 2010, ApJ, 708, 427 
Lobanov, A. P. \& Roland, J. 2005, A\& A, 431, 831

Lodato, G., King, A. R., \& Pringle, J. E. 2009, MNRAS, 392, 332

Lodato, G. \& Rossi, E. M. 2011, MNRAS, 410, 359

Lousto, C. O. \& Zlochower, Y. 2011, Physical Review Letters, 107, 231102

Luminet, J.-P. 1985, Annales de Physique, 10, 101

Maksym, W. P., Ulmer, M. P., \& Eracleous, M. 2010, ApJ, 722, 1035

Maksym, W. P., Ulmer, M. P., Eracleous, M. C., Guennou, L., \& Ho, L. C. 2013, MNRAS, 435, 1904

Mayer, L. 2013, Classical and Quantum Gravity, 30, 244008

McKernan, B., Ford, K. E. S., Kocsis, B., \& Haiman, Z. 2013, MNRAS, 432, 1468

Meiron, Y. \& Laor, A. 2013, MNRAS, 433, 2502

Merritt, D. \& Ekers, R. D. 2002, Science, 297, 1310

Merritt, D. \& Milosavljević, M. 2005, Living Reviews in Relativity, 8, 8

Mezcua, M., Chavushyan, V. H., Lobanov, A. P., \& León-Tavares, J. 2012, A\&\&A, 544, A36

Milosavljević, M. \& Phinney, E. S. 2005, ApJ, 622, L93

Murphy, D. W., Preston, R. A., \& Hirabayashi, H. 2003, New A Rev., 47, 633

Nikołajuk, M. \& Walter, R. 2013, A\& A, 552, A75

Ostorero, L., Villata, M., \& Raiteri, C. M. 2004, A\&\&A, 419, 913

Perets, H. B. \& Alexander, T. 2008, ApJ, 677, 146

Popović, L. C. 2012, New A Rev., 56, 74

Preto, M., Berentzen, I., Berczik, P., \& Spurzem, R. 2011, ApJ, 732, L26

Qian, S.-J., Kudryavtseva, N. A., Britzen, S., et al. 2007, Chinese J. Astron. Astrophys., 7, 364

Raiteri, C. M., Villata, M., Aller, H. D., et al. 2001, A\&SA, 377, 396

Rees, M. J. 1988, Nature, 333, 523

-. 1990, Science, 247,817

Rieger, F. M. 2004, ApJ, 615, L5

Rieger, F. M. \& Mannheim, K. 2000, A\&3A, 353, 473

Rödig, C., Burkart, T., Elbracht, O., \& Spanier, F. 2009, A\&A, 501, 925

Rodriguez, C., Taylor, G. B., Zavala, R. T., et al. 2006, ApJ, 646, 49

Roland, J., Britzen, S., Kun, E., et al. 2015, A\&A, 578, A86

Roos, N. 1981, $A \& A$, 104, 218

-. 1988, ApJ, 334, 95

Roos, N., Kaastra, J. S., \& Hummel, C. A. 1993, ApJ, 409, 130

Rossi, E. M., Donnarumma, I., Fender, R., et al. 2015, ArXiv e-prints

Saxton, R. D., Read, A. M., Esquej, P., et al. 2012, A\&SA, 541, A106

Schnittman, J. D. 2011, Classical and Quantum Gravity, 28, 094021

Schutz, B. F. 1986, Nature, 323, 310

Sesana, A. 2015, Astrophysics and Space Science Proceedings, 40, 147

Shen, Y., Liu, X., Greene, J. E., \& Strauss, M. A. 2011, ApJ, 735, 48

Shen, Y., Liu, X., Loeb, A., \& Tremaine, S. 2013, ApJ, 775, 49

Shen, Y. \& Loeb, A. 2010, ApJ, 725, 249

Shi, Z.-X., Luo, A.-L., Comte, G., et al. 2014, Research in Astronomy and Astrophysics, 14, 1234

Shiokawa, H., Krolik, J. H., Cheng, R. M., Piran, T., \& Noble, S. C. 2015, ApJ, 804, 85

Sillanpää, A., Haarala, S., Valtonen, M. J., Sundelius, B., \& Byrd, G. G. 1988, ApJ, 325, 628

Smith, K. L., Shields, G. A., Bonning, E. W., et al. 2010, ApJ, 716, 866

Smith, K. L., Shields, G. A., Salviander, S., Stevens, A. C., \& Rosario, D. J. 2012, ApJ, 752, 63

Storchi-Bergmann, T., Eracleous, M., Livio, M., et al. 1995, ApJ, 443, 617

Strubbe, L. E. \& Quataert, E. 2011, MNRAS, 415, 168

Sudou, H., Iguchi, S., Murata, Y., \& Taniguchi, Y. 2003, Science, 300, 1263

Tchekhovskoy, A., Metzger, B. D., Giannios, D., \& Kelley, L. Z. 2014, MNRAS, 437, 2744

Tilanus, R. P. J., Krichbaum, T. P., Zensus, J. A., et al. 2014, ArXiv e-prints, arXiv:1406.4650

Tsalmantza, P., Decarli, R., Dotti, M., \& Hogg, D. W. 2011, ApJ, 738, 20

Valtaoja, E., Teräsranta, H., Tornikoski, M., et al. 2000, ApJ, 531, 744

Valtonen, M. J. 2007, ApJ, 659, 1074 
Valtonen, M. J., Ciprini, S., \& Lehto, H. J. 2012, MNRAS, 427, 77

Valtonen, M. J., Lehto, H. J., Nilsson, K., et al. 2008, Nature, 452, 851

van Velzen, S., Farrar, G. R., Gezari, S., et al. 2011, ApJ, 741, 73

van Wassenhove, S., Volonteri, M., Mayer, L., et al. 2012, ApJ, 748, L7

Vasiliev, E. 2015, in these proceedings

Villata, M. \& Raiteri, C. M. 1999, A\& A, 347, 30

Volvach, A. E., Volvach, L. N., Larionov, M. G., Aller, H. D., \& Aller, M. F. 2007, Astronomy Reports, 51, 450

Wang, J.-M., Chen, Y.-M., Hu, C., et al. 2009, ApJ, 705, L76

Wang, T.-G., Zhou, H.-Y., Komossa, S., et al. 2012, ApJ, 749, 115

Wang, T.-G., Zhou, H.-Y., Wang, L.-F., Lu, H.-L., \& Xu, D. 2011, ApJ, 740, 85

Woo, J.-H., Cho, H., Husemann, B., et al. 2014, MNRAS, 437, 32

Wrobel, J. M., Walker, R. C., \& Fu, H. 2014, ApJ, 792, L8

Xie, G. Z., Yi, T. F., Li, H. Z., Zhou, S. B., \& Chen, L. E. 2008, AJ, 135, 2212

$\mathrm{Xu}$, D. \& Komossa, S. 2009, ApJ, 705, L20

Yu, Q. \& Lu, Y. 2001, A\&A, 377, 17

Yu, Q., Lu, Y., Mohayaee, R., \& Colin, J. 2011, ApJ, 738, 92

Yuan, W. 2015, in these proceedings

Zauderer, B. A., Berger, E., Margutti, R., et al. 2013, ApJ, 767, 152

Zauderer, B. A., Berger, E., Soderberg, A. M., et al. 2011, Nature, 476, 425

Zhou, H., Wang, T., Zhang, X., Dong, X., \& Li, C. 2004, ApJ, 604, L33 